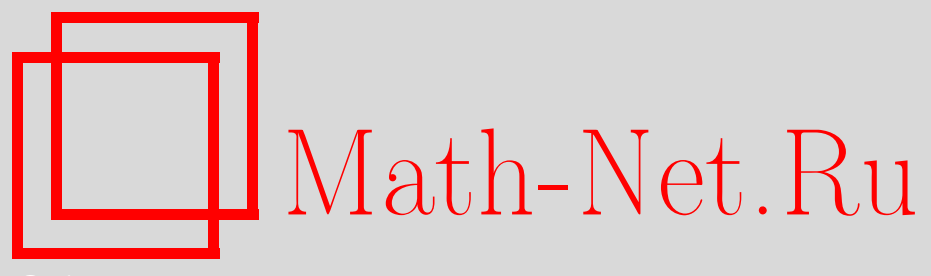

М. Г. Плотников, Об интегралах обобщенного римановского типа на плоскости и об одном примере двойного ряда Хаара, Матем. заметки, 2009, том 86, выпуск 4, 601-611

DOI: https://doi.org/10.4213/mzm4435

Использование Общероссийского математического портала Math-Net.Ru подразумевает, что вы прочитали и согласны с пользовательским соглашением http://www . mathnet.ru/rus/agreement

Параметры загрузки:

IP: 54.164 .48 .24

26 апреля 2023 г., $11: 58: 55$

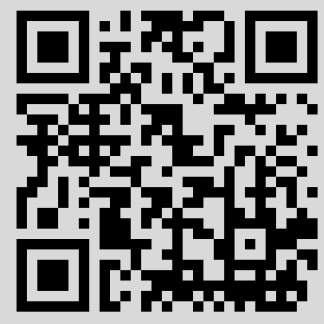


Том 86 выпуск 4 октябрь 2009

\section{Об интегралах обобщенного римановского типа на плоскости и об одном примере двойного ряда Хаара}

\section{М. Г. Плотников}

Доказывается, что для двойных рядов Хаара, для двоичного кубического интеграла Хенстока и для регулярной сходимости по прямоугольникам не выполняется теорема типа Дю Буа-Реймона.

Библиография: 12 названий.

Классическая теорема Дю Буа-Реймона $[1 ;$ гл. $1, \S 72]$ утверждает, что тригонометрический ряд $a_{0} / 2+\sum_{n=1}^{\infty} a_{n} \cos n x+b_{n} \sin n x$, всюду сходящийся к интегрируемой по Риману функции $f(x)$, является рядом Фурье этой функции, т.е.

$$
\begin{aligned}
& a_{n}=\frac{1}{\pi} \int_{-\pi}^{\pi} f(x) \cos n x d x, \quad n=0,1, \ldots, \\
& b_{n}=\frac{1}{\pi} \int_{-\pi}^{\pi} f(x) \sin n x d x, \quad n=1, \ldots .
\end{aligned}
$$

Этот результат обобщался на случай других интегралов и переносился на ряды по различным системам функций. В [2; теорема 6$]$ подобный результат был доказан для двойных рядов Хаара

$$
(S)=\sum_{n=1}^{\infty} \sum_{m=1}^{\infty} a_{n, m} \chi_{n, m}(x, y)
$$

и для $\left(H_{1 / 2, \mathrm{~d}}\right)$-интеграла (см. определение 3 ниже). Мы покажем, что для интеграла, чуть более общего, чем $\left(H_{1 / 2, \mathrm{~d}}\right)$-интеграл, теорема типа Дю Буа-Реймона для рядов (1) перестает выполняться.

Символом $A^{\circ}$ обозначим внутренность множества $A$ в топологии $\mathbb{R}^{2}$. Под мерой в работе понимается мера Лебега в $\mathbb{R}^{2}$. Пусть $\|\cdot\|-$ евклидова норма в $\mathbb{R}^{2}$. Тогда для $\mathbf{z} \in \mathbb{R}^{2}$ и $F \subset \mathbb{R}^{2}$ выражение $\operatorname{dist}(\mathbf{z}, F)$ будет означать $\inf _{\mathbf{y} \in F}\|\mathbf{z}-\mathbf{y}\|$, а выражение $\operatorname{diam} F-$ диаметр множества $F$, т.е. $\sup _{\mathbf{y}_{1}, \mathbf{y}_{2} \in F}\left\|\mathbf{y}_{1}-\mathbf{y}_{2}\right\|$. Символом $B(\mathbf{z}, r)$ обозначим открытый шар из $\mathbb{R}^{2}$ радиуса $r$ с центром в точке $\mathbf{z}$. Два множества $J_{1}, J_{2} \subset \mathbb{R}^{2}$ называются неперекрывающимися, если $J_{1}^{\circ} \cap J_{2}^{\circ}=\varnothing$. Выражение $(R) \int_{\Delta} f$ означает интеграл в смысле Римана от функции $f$ по множеству $\Delta \subset \mathbb{R}^{2}$.

Работа выполнена при поддержке Российского фонда фундаментальных исследований (грант № 08-01-00669), программы "Ведущие научные школы" (грант № НШ-4681.2006.1) и программы "Молодые ученые" (грант № MK-3667.2007.1).

(C) М. Г. Плотников, 2009 
Точки множества $Q_{\mathrm{d}}:=\left\{p / 2^{n} \in[0,1]: p, n \in \mathbb{Z}_{+}\right\}$называются двоично-рациональными, а точки множества $I_{\mathrm{d}}:=[0,1] \backslash Q_{\mathrm{d}}-$ двоично-иррачиональными. Двоичный интервал ранга $(k, l)$ есть замкнутый сегмент вида

$$
\Delta_{k, l}^{i, j}=\left[\frac{i-1}{2^{k}} ; \frac{i}{2^{k}}\right] \times\left[\frac{j-1}{2^{l}} ; \frac{j}{2^{l}}\right]
$$

$k, l=0,1, \ldots, i=1, \ldots, 2^{k}, j=1, \ldots, 2^{l}$. Двоичный интервал (2) с $k=l$ называется двоичным квадратом. Пусть $\mathbf{z} \in[0,1]^{2}$. Двойную последовательность $\left\{\Delta_{k, l}\right\}_{k, l=0}^{\infty}$ двоичных интервалов назовем $\mathbf{z}$-последовательностью, если для всех $k, l=0,1, \ldots$ выполнены следующие условия: $\mathbf{z} \in \Delta_{k, l}$, ранг двоичного интервала $\Delta_{k, l}$ равен $(k, l)$, $\Delta_{k+1, l} \subset \Delta_{k, l}, \Delta_{k, l+1} \subset \Delta_{k, l}$.

Пусть $\phi(x)$ есть 1 при $x \in(0,1 / 2),-1$ при $x \in(1 / 2,1)$ и 0 при $x \notin[0,1]$. Тогда $c u$ стема Хаара $\left\{\chi_{n}(x)\right\}_{n=1}^{\infty}, x \in[0,1]$, определяется так: $\chi_{1}(x) \equiv 1$; если же $n=2^{k}+i$, $k=0,1, \ldots, 0 \leqslant i \leqslant 2^{k}-1$, то $\chi_{n+1}(x)=2^{k / 2} \phi\left(2^{k} x-i\right)$. В точке 0 (соответственно 1 ) функция $\chi_{n}(x)$ равна своему пределу справа (соответственно слева), а в остальных точках отрезка $[0,1]$ - среднему арифметическому правого и левого пределов. При таком определении ряд Фурье-Хаара любой функции из $C[0,1]$ сходится к ней равномерно на $[0,1]$ (см. [3; гл. $3, \S 2],[4])$.

Двумерная функция Хаара $\chi_{n, m}(x, y)$ есть $\chi_{n}(x) \chi_{m}(y)$. Выражение $S_{N, M}(x, y):=$ $\sum_{n=1}^{N} \sum_{m=1}^{M} a_{n, m} \chi_{n, m}(x, y)$ означает прямоугольную частичную сумму ряда (1) в точке $(x, y)$. Параметром регулярности двоичного интервала $\Delta$ назовем число $\operatorname{reg} \Delta$, равное отношению длин минимальной и максимальной сторон этого интервала, а параметром регулярности вектора $\mathbf{Q}=\left(Q_{1}, Q_{2}\right)$ - число $\operatorname{reg} \mathbf{Q}=\operatorname{reg}\left(Q_{1}, Q_{2}\right)$, равное $\min \left\{Q_{1} / Q_{2}, Q_{2} / Q_{1}\right\}$. Пусть $\rho \in(0,1]$. Тогда ряд (1) $\rho$-сходится в точке $(x, y)$ к значению $S(x, y)$, если

$$
S_{N, M}(x, y) \rightarrow S(x, y), \quad \min \{N, M\} \rightarrow \infty, \quad \operatorname{reg}(N, M) \geqslant \rho .
$$

Ряд (1) сходится по прямоуголъникам в точке $(x, y)$ к значению $S(x, y)$, если

$$
S_{N, M}(x, y) \rightarrow S(x, y), \quad \min \{N, M\} \rightarrow \infty .
$$

Ясно, что из сходимости по прямоугольникам ряда $(1)$ в точке $(x, y)$ вытекает $\rho$-сходимость этого ряда в точке $(x, y)$ при любом $\rho \in(0,1]$.

Пусть $\mathscr{I}$ означает множество всех двоичных интервалов. Функции множества $\Phi: \mathscr{I} \rightarrow \mathbb{R}$ будем называть $\mathscr{I}$-функциями. $\mathscr{I}$-Функция $\Phi$ называется супераддитивной (соответственно субаддитивной), если

$$
\sum_{i=1}^{p} \Phi\left(\Delta_{i}\right) \leqslant \Phi\left(\bigcup_{i=1}^{p} \Delta_{i}\right) \quad\left(\sum_{i=1}^{p} \Phi\left(\Delta_{i}\right) \geqslant \Phi\left(\text { соответственно } \bigcup_{i=1}^{p} \Delta_{i}\right)\right)
$$

где $\Delta_{i} \in \mathscr{I}, i=1, \ldots, p, \bigcup_{i=1}^{p} \Delta_{i} \in \mathscr{I}$ и $\Delta_{i}^{\circ} \cap \Delta_{j}^{\circ}=\varnothing$, если $i \neq j$. Пусть $\overline{\mathscr{A}}_{\mathscr{I}}$ (соответственно $\mathscr{A}_{\mathscr{I}}$ ) означает класс супераддитивных (соответственно суббадитивных) $\mathscr{I}$-функций. $\mathscr{I}$-Функции из класса $\mathscr{A}_{\mathscr{I}}:=\overline{\mathscr{A}}_{\mathscr{I}} \cap \mathscr{A}_{\mathscr{I}}$ называются аддитивными, а в ряде работ (см., например, [5]) их называют квазимерами.

Имея ряд (1), можно определить $\mathscr{I}$-функцию $\Psi$ формулой

$$
\Psi\left(\Delta_{k, l}\right)=S_{2^{k}, 2^{l}}(\mathbf{z})\left|\Delta_{k, l}\right|,
$$


где $\Delta_{k, l}$ - двоичный интервал ранга $(k, l), \mathbf{z}$ - любая его внутренняя точка, a $\left|\Delta_{k, l}\right|-$ его площадь. При этом значение $\Psi\left(\Delta_{k, l}\right)$, определяемое формулой $(3)$, не зависит от $\mathbf{z} \in \Delta_{k, l}$. Хорошо известно (см., например, [6]), что функция $\Psi$ является квазимерой. При этом соответствие между рядами (1) и квазимерами, определяемое формулой (3), является линейным изоморфизмом, если естественным образом наделить множество рядов (1) и множество квазимер структурой линейного пространства.

Теорема 1. Пусть заданы $\rho \in(0,1]$, ряд (1) и точка $\mathbf{z} \in[0,1]^{2}$. Пусть $\Psi-$ $\mathscr{I}$-функиия, построенная для ряда (1) по формуле (3). Если для этой функиии, для любой $\mathbf{z}$-последовательности $\left\{\Delta_{k, l}\right\}$ и для некоторого $A \in \mathbb{R}$ выполняется равенство

$$
\Psi\left(\Delta_{k, l}\right)=A\left|\Delta_{k, l}\right|+\overline{\bar{o}}\left(\left|\Delta_{k, l}\right|\right), \quad \min \{k, l\} \rightarrow \infty, \quad \operatorname{reg}\left(2^{k}, 2^{l}\right) \geqslant \frac{\rho}{2},
$$

то ряд (1) $\rho$-сходится в точке $\mathbf{z} \kappa$ значению $A$.

ДокАЗАтЕЛЬСтво. Будем считать, что $x, y \notin\{0,1\}$ (в противном случае доказательство аналогично). Одномерные функции Хаара $\chi_{n}(x)$ обладают тем свойством, что в любой точке $x \in(0,1)$ при достаточно малом $\varepsilon=\varepsilon(n)>0$ имеет место равенство $\chi_{n}(x)=\left(\chi_{n}(x-\varepsilon)+\chi_{n}(x+\varepsilon)\right) / 2$. Тогда для двумерных функций Хаара $\chi_{n, m}$ при достаточно малом $\varepsilon>0$ выполнено равенство

$$
\chi_{n, m}(x, y)=\frac{1}{4} \sum_{\alpha, \beta=0,1} \chi_{n, m}\left(x+(-1)^{\alpha} \varepsilon, y+(-1)^{\beta} \varepsilon\right) .
$$

Из (5) вытекает, что для частичных сумм $S_{N, M}(x, y)$ ряда (1) при достаточно малом $\varepsilon>0$ выполнено равенство

$$
S_{N, M}(x, y)=\frac{1}{4} \sum_{\alpha, \beta=0,1} S_{N, M}\left(x+(-1)^{\alpha} \varepsilon, y+(-1)^{\beta} \varepsilon\right) .
$$

Из структуры ряда (1) следует, что

$$
S_{N, M}\left(x+(-1)^{\alpha} \varepsilon, y+(-1)^{\beta} \varepsilon\right)=S_{2^{k}, 2^{l}}\left(x+(-1)^{\alpha} \varepsilon, y+(-1)^{\beta} \varepsilon\right),
$$

где $k=k(\alpha, N, x, \varepsilon)$ и $l=l(\beta, M, y, \varepsilon)$ таковы, что $\operatorname{reg}\left(2^{k}, 2^{l}\right) \geqslant \rho / 2$. Используя (3), $(4),(6)$ и (7), получаем

$$
\begin{aligned}
S_{N, M}(x, y) & =\frac{1}{4} \sum_{\alpha, \beta=0,1} S_{2^{k}, 2^{l}}\left(x+(-1)^{\alpha} \varepsilon, y+(-1)^{\beta} \varepsilon\right)=\frac{1}{4} \sum_{\alpha, \beta=0,1} \frac{\Psi\left(\Delta_{k, l}^{(\alpha, \beta)}\right)}{\left|\Delta_{k, l}^{(\alpha, \beta)}\right|} \\
& =\frac{1}{4} \sum_{\alpha, \beta=0,1}(A+\overline{\bar{o}}(1))=A+\overline{\bar{o}}(1), \quad \min \{k, l\} \rightarrow \infty, \quad \operatorname{reg}\left(2^{k}, 2^{l}\right) \geqslant \frac{\rho}{2},
\end{aligned}
$$

где $\Delta_{k, l}^{(\alpha, \beta)}$ - двоичный интервал из одной из $(x, y)$-последовательностей, содержащий точку $\left(x+(-1)^{\alpha} \varepsilon, y+(-1)^{\beta} \varepsilon\right)$. Последняя цепочка доказывает теорему.

Рассмотрим при $\rho=1 / 2$ один обобщенный интеграл, построенный в [7] для решения проблемы восстановления коэффициентов рядов (1). 
ОПРЕДЕЛЕНиЕ 1. Скажем, что функция $f(x, y):\left(I_{\mathrm{d}}\right)^{2} \rightarrow \mathbb{R}$ является $\left(P_{\mathrm{d}}^{1 / 2}\right)$-интегрируемой, если для любого $\varepsilon>0$ найдутся $\mathscr{I}$-функции $F_{1} \in \overline{\mathscr{A}}_{\mathscr{I}}$ (мажоранта) и $F_{2} \in \mathscr{A}_{\mathscr{I}}$ (миноранта) такие, что

1) если $\mathbf{z} \in\left(I_{\mathrm{d}}\right)^{2}$ и $\left\{\Delta_{k, l}\right\}$ является $\mathbf{z}$-последовательностью, то

$$
\varliminf_{n \rightarrow \infty} \frac{F_{1}\left(\Delta_{n, n}\right)}{\left|\Delta_{n, n}\right|} \geqslant f(\mathbf{z}) \geqslant \varlimsup_{n \rightarrow \infty} \frac{F_{2}\left(\Delta_{n, n}\right)}{\left|\Delta_{n, n}\right|}
$$

2) если $\mathbf{z} \in Q_{\mathrm{d}} \times I_{\mathrm{d}}, \mathbf{z} \in I_{\mathrm{d}} \times Q_{\mathrm{d}}$ или $\mathbf{z} \in Q_{\mathrm{d}} \times Q_{\mathrm{d}}$ и $\left\{\Delta_{k, l}\right\}$ является любой из $\mathbf{z}$-последовательностей, то для $i=1,2$

$$
\begin{aligned}
& \lim _{n \rightarrow \infty} \frac{1}{\left|\Delta_{n, n}\right|}\left[F_{i}\left(\Delta_{n, n}\right)-\frac{1}{2} F_{i}\left(\Delta_{n-1, n}\right)\right]=0, \\
& \lim _{n \rightarrow \infty} \frac{1}{\left|\Delta_{n, n}\right|}\left[F_{i}\left(\Delta_{n, n}\right)-\frac{1}{2} F_{i}\left(\Delta_{n, n-1}\right)\right]=0
\end{aligned}
$$

или

$$
\lim _{n \rightarrow \infty} \frac{F_{i}\left(\Delta_{n, n}\right)-F_{i}\left(\Delta_{n, n-1}\right) / 2-F_{i}\left(\Delta_{n-1, n}\right) / 2+F_{i}\left(\Delta_{n-1, n-1}\right) / 4}{\left|\Delta_{n, n}\right|}=0
$$

соответственно;

3) $F_{1}\left([0,1]^{2}\right)-F_{2}\left([0,1]^{2}\right)<\varepsilon$.

$\left(P_{\mathrm{d}}^{1 / 2}\right)$-Интеграл от функции $f(x, y)$ определяется для любого двоичного интервала $\Delta$ так:

$$
\left(P_{d}^{1 / 2}\right) \int_{\Delta} f(x, y) d x d y=\sup _{F_{2}} F_{2}(\Delta)=\inf _{F_{1}} F_{1}(\Delta) .
$$

Tеорема A [7; теорема 2]. Пусть для некоторого $\rho \in(0,1 / 2]$ ряд (1) всюду на $[0,1]^{2} \quad \rho$-сходится $\kappa$ конечной функции $f(x, y)$. Тогда функиия $f(x, y)$ является $\left(P_{\mathrm{d}}^{1 / 2}\right)$-интегрируемой и данный ряд есть ряд Фуръе-Хаара относительно $\left(P_{\mathrm{d}}^{1 / 2}\right)$-интеграла, т.е. его коэффициенты определяются формулой

$$
a_{n, m}=\left(P_{\mathrm{d}}^{1 / 2}\right) \int_{[0,1]^{2}} f(x, y) \chi_{n, m}(x, y) d x d y
$$

ОПРеДЕЛЕНИЕ 2. Пусть задано $\rho \in(0,1]$. Разбиением двоичного интервала $I_{0}$ называется набор $P=\left\{\left(\mathbf{z}_{j}, I_{j}\right)\right\}_{j=1}^{m}$, где $I_{j}$ - двоичные интервалы, причем $I_{j}^{\circ} \cap I_{k}^{\circ}=\varnothing$, если $j \neq k, \mathbf{z}_{j} \in I_{0}$ и $\bigcup_{j=1}^{m} I_{j}=I_{0}$. Пусть задана функция $\delta(\mathbf{z}): I_{0} \rightarrow(0 ;+\infty)$. Тогда разбиение $P$ называется $(\delta(\mathbf{z}), \rho)$-разбиением, если $\mathbf{z}_{j} \in I_{j} \subset B\left(\mathbf{z}_{j}, \delta\left(\mathbf{z}_{j}\right)\right)$ и reg $I_{j} \geqslant \rho$ для всех $j=1, \ldots, m$.

ОПРЕДЕЛЕНИЕ 3 . Пусть $\rho \in(0,1]$, а $\Delta$ - двоичный интервал. Функция $f: \Delta \rightarrow \mathbb{R}$ называется интегрируемой по Хенстоку в $\rho$-регулярном двоичном смысле $\left(\left(H_{\rho, \mathrm{d}}\right)\right.$ интегрируемой) на интервале $\Delta$, если существует действительное $A$, и для любого $\varepsilon>0$ существует функция $\delta: \Delta \rightarrow(0,+\infty)$ такая, что для всех $(\delta(\mathbf{z}), \rho)$-разбиений $P=\left\{\left(\mathbf{z}_{j} ; I_{j}\right)\right\}_{j=1}^{m}$ интервала $\Delta$ выполняется неравенство $\left|\sum_{j=1}^{m} f\left(\mathbf{z}_{j}\right)\right| I_{j}|-A|<\varepsilon$. При этом $A=\left(H_{\rho, \mathrm{d}}\right) \int_{\Delta} f(x, y) d x d y$. 
Если функция $f$ является $\left(H_{\rho, \mathrm{d}}\right)$-интегрируемой на двоичных интервалах $\Delta_{1}$ и $\Delta_{2}$, то она является $\left(H_{\rho, \mathrm{d}}\right)$-интегрируемой и на $\Delta_{1} \cup \Delta_{2}$. Если функция $f$ является $\left(H_{\rho, \mathrm{d}}\right)$-интегрируемой на $[0,1]^{2}$, то $\mathscr{I}$-функция

$$
F(\Delta)=\left(H_{\rho, \mathrm{d}}\right) \int_{\Delta} f(x, y) d x d y
$$

является квазимерой (см. [8]). Отметим следующий факт, вытекающий из определений 2 и 3.

УтВеРЖДЕНИЕ 1. Если функиия $f: \Delta \rightarrow \mathbb{R}$ является непрерывной на двоичном интервале $\Delta$, то она является $\left(H_{\rho, \mathrm{d}}\right)$-интегрируемой на $\Delta$, причем

$$
\left(H_{\rho, \mathrm{d}}\right) \int_{\Delta} f(x, y) d x d y=(R) \int_{\Delta} f(x, y) d x d y .
$$

ОПРЕДЕЛЕНИЕ 4. Пусть $C_{1}$ и $C_{2}$ - классы интегрируемых в каком-либо смысле функций. Соответствующие интегралы называются непротиворечивыми, если $\left(C_{1}\right) \int_{\Delta} f=\left(C_{2}\right) \int_{\Delta} f$ для всех допустимых множеств $\Delta$ и для всех $f \in C_{1} \cap C_{2}$.

В [2] было доказано, что $\left(P_{\mathrm{d}}^{1 / 2}\right)$-интеграл и $\left(H_{1 / 2, \mathrm{~d}}\right)$-интеграл являются непротиворечивыми. Следствием этого факта и теоремы А являлся следующий результат.

Теорема В [2; теорема 7]. Пусть задано $\rho \in(0,1 / 2]$. Если ряд (1) всюду на $[0,1]^{2}$ р-сходится к конечной функиии $f(\mathbf{z})$, являющейся $\left(H_{1 / 2, \mathrm{~d}}\right)$-интегрируемой, то данный ряд является рядом Фуръе-Хаара функции $f(\mathbf{z})$ относительно $\left(H_{1 / 2, \mathrm{~d}}\right)$ интеграла.

Теорема В является теоремой типа Дю Буа-Реймона и обобщает известную теорему Скворцова (см. [9]). Мы покажем, что теорема В в некотором смысле неусиливаема. То, что в теореме В вместо $\rho \in(0,1 / 2]$ нельзя рассмотреть значения $\rho$, близкие к единице, показывают результаты работы [10], где для каждого $\rho \in(\sqrt{2} / 2,1]$ был построен тождественно ненулевой ряд (1), $\rho$-сходящийся к нулю всюду на $[0,1]^{2}$. Мы докажем, что теорема В перестает быть верной, если заменить в ней $\left(H_{1 / 2, \mathrm{~d}}\right)$-интеграл на $\left(H_{1, \mathrm{~d}}\right)$-интеграл. Параллельно будет установлена противоречивость $\left(H_{1 / 2, \mathrm{~d}}\right)$ интеграла и $\left(P_{\mathrm{d}}^{1 / 2}\right)$-интеграла. Основной в работе является

Конструкция 1 . Построим квазимеру $\Psi$ и функцию $f:[0,1]^{2} \rightarrow \mathbb{R}$. До конца работы $\widetilde{z}$ означает точку $(1 / 2,1 / 2)$. Функцию $\Psi$ определим сначала на двоичных квадратах. Положим

$$
\Psi\left([0,1]^{2}\right)=0
$$

Рассмотрим двоичные квадраты $\Delta_{n, n}^{i, j}$ в нумерации (2). Пусть $\Omega_{1}=\Delta_{1,1}^{1,1}, \Omega_{2}=\Delta_{1,1}^{1,2}$, $\Omega_{3}=\Delta_{1,1}^{2,2}$ и $\Omega_{4}=\Delta_{1,1}^{2,1}$. Положим

$$
\Psi\left(\Omega_{1}\right)=\Psi\left(\Omega_{3}\right)=1, \quad \Psi\left(\Omega_{2}\right)=\Psi\left(\Omega_{4}\right)=-1 .
$$

Рассмотрим теперь двоичные квадраты $\Delta_{n, n}^{i, j}$, лежащие в $\Omega_{1}$. При $n=2,3, \ldots$ положим

$$
\begin{gathered}
\Psi\left(\Delta_{n, n}^{2^{n-1}-1,2^{n-1}}\right)=\Psi\left(\Delta_{n, n}^{2^{n-1}, 2^{n-1}-1}\right)=\frac{1}{4}, \quad \Psi\left(\Delta_{n, n}^{2^{n-1}, 2^{n-1}}\right)=1 \\
\Psi\left(\Delta_{n, n}^{2^{n-1}-1,2^{n-1}-1}\right)=-\frac{1}{2}
\end{gathered}
$$


Пусть $n=3,4, \ldots$. При $s \in\left\{2^{n-1}-2,2^{n-1}-3\right\}, t=2^{n-1}$ и $v=2^{n-1}-1$ положим

$$
\Psi\left(\Delta_{n, n}^{s, t}\right)=\Psi\left(\Delta_{n, n}^{t, s}\right)=\frac{7}{16}, \quad \Psi\left(\Delta_{n, n}^{s, v}\right)=\Psi\left(\Delta_{n, n}^{v, s}\right)=-\frac{5}{16} .
$$

Возьмем любой двоичный квадрат $\Delta \subset \Omega_{1}$, не совпадающий ни с одним квадратом из левых частей формул (14)-(17). Отметим, что в этом случае $\widetilde{\mathbf{z}} \notin \Delta$. Кроме того, из геометрических соображений вытекает, что $\Delta \subset J$, где $J$ - единственным образом определяемый двоичный квадрат, участвующий в одной из формул (16) или (17). Для каждого такого квадрата $J$ выберем произвольную непрерывную функцию $f(x, y): J \rightarrow \mathbb{R}$, удовлетворяющую следующим свойствам:

- $(R) \int_{J} f(x, y) d x d y=\Psi(J)$;

- $\operatorname{supp} f \subset J^{\circ}$.

В результате функция $f(x, y)$ оказывается определенной и непрерывной на множестве $\Omega_{1} \backslash\{\widetilde{\mathbf{z}}\}$. Для всех двоичных квадратов $\Delta \subset\left(\Omega_{1} \backslash\{\widetilde{\mathbf{z}}\}\right)$ положим

$$
\Psi(\Delta)=(R) \int_{\Delta} f(x, y) d x d y
$$

Формулы (14)-(18) определяют функцию $\Psi$ на всех двоичных квадратах $\Delta \subset \Omega_{1}$. Если двоичный квадрат $\Delta_{n, n}^{i, j}, n \geqslant 1$, лежит в $\Omega_{2} \cup \Omega_{3} \cup \Omega_{4}$, то значение $\Psi\left(\Delta_{n, n}^{i, j}\right)$ определяется по значению функции $\Psi$ на некотором двоичном квадрате $\Delta \subset \Omega_{1}$ следующим образом:

$$
\Psi\left(\Delta_{n, n}^{i, j}\right)= \begin{cases}-\Psi\left(\Delta_{n, n}^{i, 2^{n}-j+1}\right) & \text { при } 1 \leqslant i \leqslant 2^{n-1}, 2^{n-1}+1 \leqslant j \leqslant 2^{n} ; \\ \Psi\left(\Delta_{n, n}^{2^{n}-i+1,2^{n}-j+1}\right) & \text { при } 1 \leqslant i, j \leqslant 2^{n-1} ; \\ -\Psi\left(\Delta_{n, n}^{2^{n}-i+1, j}\right) & \text { при } 2^{n-1}+1 \leqslant i \leqslant 2^{n}, 1 \leqslant j \leqslant 2^{n-1} .\end{cases}
$$

Тем самым $\mathscr{I}$-функция $\Psi$ определена на всех двоичных квадратах. Из (13)-(19) вытекает аддитивность функции $\Psi$ на двоичных квадратах. Если в определениях кольца и полукольца множеств рассматривать вместо непересекающихся множеств неперекрывающиеся, то семейство двоичных квадратов является полукольцом, которое порождает кольцо, состоящее из конечных объединений двоичных интервалов. По теореме о продолжении меры с полукольца на порождаемое им кольцо (см., например, [11; гл. 5]) функция $\Psi$ единственным образом продолжается до квазимеры.

Рассмотрим теперь функцию $f(x, y)$, определенную на множестве $\Omega_{1} \backslash\{\widetilde{\mathbf{z}}\}$. Распространим эту функцию на множество $[0,1]^{2}$, полагая для всех $0 \leqslant x, y \leqslant 1$

$$
f(x, y)=f(1-x, 1-y)=-f(1-x, y)=-f(x, 1-y) .
$$

Функция $f$ конечна на $[0,1]^{2}$, непрерывна на $[0,1]^{2} \backslash\{\widetilde{\mathbf{z}}\}$ и $f(1 / 2,1 / 2)=0$.

ЗАмЕчАниЕ 1. В силу (19) и (20) равенство (18) справедливо для всех двоичных квадратов, не содержащих точку $\widetilde{\mathbf{z}}$.

Далее мы рассмотрим ряд $\left(S^{\star}\right)$ вида $(1)$, который изоморфен при помощи формулы (3) функции $\Psi$ из конструкции 1.

Лемма 1. Всюду на $[0,1]^{2}$ ряд $\left(S^{\star}\right)(1 / 2)$-сходится $\kappa$ функиии $f$ из конструкu,uи 1. 
ДоказатеЛЬСтво. Пусть $(x, y) \neq(1 / 2,1 / 2)$. В силу замечания 1 равенство (18) справедливо для всех достаточно малых по диаметру двоичных интервалов, содержащих точку $(x, y)$. С учетом непрерывности функции $f$ на $[0,1]^{2} \backslash\{\widetilde{\mathbf{z}}\}$ из (18) получаем

$$
\Psi(\Delta)=f(x, y)|\Delta|+\overline{\bar{o}}(|\Delta|), \quad \operatorname{diam} \Delta \rightarrow 0, \quad(x, y) \in \Delta .
$$

Из (21) и из теоремы 1 вытекает утверждение леммы для точек $(x, y) \neq(1 / 2,1 / 2)$.

Рассмотрим теперь частичные суммы $S_{N, M}(1 / 2,1 / 2)$ ряда $\left(S^{\star}\right)$ и покажем, что

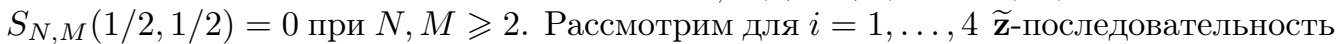
$\left\{\Delta_{k, l}^{i}\right\}$, каждый интервал из которой лежит при $k, l \geqslant 1$ в двоичном квадрате $\Omega_{i}$. Из (15), (16) и (18) и из аддитивности функции $\Psi$ вытекает справедливость при $n \geqslant 2$ равенств

$$
\begin{gathered}
\Psi\left(\Delta_{n, n-1}^{i}\right)=\Psi\left(\Delta_{n-1, n}^{i}\right)=(-1)^{i-1} \frac{5}{4}, \quad \Psi\left(\Delta_{n, n}^{i}\right)=(-1)^{i-1}, \\
\Psi\left(\Delta_{n+1, n-1}^{i}\right)=\Psi\left(\Delta_{n-1, n+1}^{i}\right)=(-1)^{i-1} \frac{17}{8} .
\end{gathered}
$$

Используя (3), (6), (7), (22) и структуру рядов (1), получаем при $n \geqslant 1$ следующие цепочки:

$$
\begin{aligned}
& S_{2^{n}, 2^{n}}\left(\frac{1}{2}, \frac{1}{2}\right)=\frac{1}{4} \sum_{\alpha, \beta=0,1} S_{2^{n}, 2^{n}}\left(\frac{1}{2}+(-1)^{\alpha} \varepsilon, \frac{1}{2}+(-1)^{\beta} \varepsilon\right) \\
& =\frac{1}{4} \sum_{i=1}^{4} \frac{\Psi\left(\Delta_{n, n}^{i}\right)}{\left|\Delta_{n, n}^{i}\right|}=\frac{1}{4\left|\Delta_{n, n}^{1}\right|} \sum_{i=1}^{4}(-1)^{i-1}=0, \\
& S_{2^{n+1}, 2^{n}}\left(\frac{1}{2}, \frac{1}{2}\right)=\frac{1}{4} \sum_{i=1}^{4} \frac{\Psi\left(\Delta_{n+1, n}^{i}\right)}{\left|\Delta_{n+1, n}^{i}\right|}=\frac{1}{4\left|\Delta_{n+1, n}^{1}\right|} \sum_{i=1}^{4}(-1)^{i-1} \frac{5}{4}=0, \\
& S_{2^{n}+2^{n-1}, 2^{n}}\left(\frac{1}{2}, \frac{1}{2}\right)=\frac{1}{4} \sum_{\alpha, \beta=0,1} S_{2^{n}+2^{n-1}, 2^{n}}\left(\frac{1}{2}+(-1)^{\alpha} \varepsilon, \frac{1}{2}+(-1)^{\beta} \varepsilon\right) \\
& =\frac{1}{4}\left(\frac{\Psi\left(\Delta_{n+1, n}^{1}\right)}{\left|\Delta_{n+1, n}^{1}\right|}+\frac{\Psi\left(\Delta_{n+1, n}^{2}\right)}{\left|\Delta_{n+1, n}^{2}\right|}+\frac{\Psi\left(\Delta_{n, n}^{3}\right)}{\left|\Delta_{n, n}^{3}\right|}+\frac{\Psi\left(\Delta_{n, n}^{4}\right)}{\left|\Delta_{n, n}^{4}\right|}\right) \\
& =\frac{1}{4\left|\Delta_{n+1, n}^{1}\right|}\left(\frac{5}{4}-\frac{5}{4}+\frac{1}{2}-\frac{1}{2}\right)=0 \text {, } \\
& S_{2^{n}+2^{n-1}, 2^{n+1}}\left(\frac{1}{2}, \frac{1}{2}\right)=\frac{1}{4}\left(\frac{\Psi\left(\Delta_{n+1, n+1}^{1}\right)}{\left|\Delta_{n+1, n+1}^{1}\right|}+\frac{\Psi\left(\Delta_{n+1, n+1}^{2}\right)}{\left|\Delta_{n+1, n+1}^{2}\right|}+\frac{\Psi\left(\Delta_{n, n+1}^{3}\right)}{\left|\Delta_{n, n+1}^{3}\right|}+\frac{\Psi\left(\Delta_{n, n+1}^{4}\right)}{\left|\Delta_{n, n+1}^{4}\right|}\right) \\
& =\frac{1}{4\left|\Delta_{n+1, n+1}^{1}\right|}\left(1-1+\frac{1}{2} \frac{5}{4}-\frac{1}{2} \frac{5}{4}\right)=0 \text {. }
\end{aligned}
$$

Пусть $l=2^{n}+2^{n-1}$. Снова используя (3), (6), (7), (22) и структуру рядов (1), получаем при $n \geqslant 1$

$$
\begin{aligned}
S_{l, l}\left(\frac{1}{2}, \frac{1}{2}\right) & =\frac{1}{4} \sum_{\alpha, \beta=0,1} S_{l, l}\left(\frac{1}{2}+(-1)^{\alpha} \varepsilon, \frac{1}{2}+(-1)^{\beta} \varepsilon\right) \\
& =\frac{1}{4}\left(\frac{\Psi\left(\Delta_{n+1, n+1}^{1}\right)}{\left|\Delta_{n+1, n+1}^{1}\right|}+\frac{\Psi\left(\Delta_{n+1, n}^{2}\right)}{\left|\Delta_{n+1, n}^{2}\right|}+\frac{\Psi\left(\Delta_{n, n}^{3}\right)}{\left|\Delta_{n, n}^{3}\right|}+\frac{\Psi\left(\Delta_{n, n+1}^{4}\right)}{\left|\Delta_{n, n+1}^{4}\right|}\right)
\end{aligned}
$$




$$
=\frac{1}{4\left|\Delta_{n+1, n+1}^{1}\right|}\left(1-\frac{1}{2} \frac{5}{4}+\frac{1}{4}-\frac{1}{2} \frac{5}{4}\right)=0 .
$$

Используя (3), (6), (7), (22), (23) и структуру рядов (1), аналогично (28) при $n \geqslant 1$ получаем

$$
\begin{aligned}
S_{2^{n+1}}+2^{n}, 2^{n}+2^{n-1} & \left(\frac{1}{2}, \frac{1}{2}\right) \\
& =\frac{1}{4}\left(\frac{\Psi\left(\Delta_{n+2, n+1}^{1}\right)}{\left|\Delta_{n+2, n+1}^{1}\right|}+\frac{\Psi\left(\Delta_{n+2, n}^{2}\right)}{\left|\Delta_{n+2, n}^{2}\right|}+\frac{\Psi\left(\Delta_{n+1, n}^{3}\right)}{\left|\Delta_{n+1, n}^{3}\right|}+\frac{\Psi\left(\Delta_{n+1, n+1}^{4}\right)}{\left|\Delta_{n+1, n+1}^{4}\right|}\right) \\
& =\frac{1}{4\left|\Delta_{n+2, n+1}^{1}\right|}\left(\frac{5}{4}-\frac{1}{2} \frac{17}{8}+\frac{1}{4} \frac{5}{4}-\frac{1}{2}\right)=0 .
\end{aligned}
$$

Пусть $N, M \geqslant 2$. Рассмотрим произвольную частичную сумму $S:=S_{N, M}(1 / 2,1 / 2)$ с $\operatorname{reg}(N, M) \geqslant 1 / 2$. Тогда имеет место следующая альтернатива. Либо $S$ совпадает с одной из частичных сумм из левых частей формул (24)-(29), в этом случае $S=0$. Либо $S$ получается из одной из частичных сумм в левых частях формул (24)-(29) заменой нижних индексов. В этом случае равенство $S=0$ доказывается аналогично тому, как это делалось в (24)-(29). В любом случае $S=f(1 / 2,1 / 2)=0$, что окончательно доказывает лемму.

ЛЕмма 2. 1) Функиия $f(x, y)$ из конструкиии 1 является $\left(P_{\mathrm{d}}^{1 / 2}\right)$-интегрируемой и ряд (1) является ее рядом Фуръе-Хаара относительно $\left(P_{\mathrm{d}}^{1 / 2}\right)$-интеграла;

2) если $\Psi-\mathscr{I}$-функиия из конструкиии 1, то для любого двоичного интервала $\Delta$ имеет место равенство

$$
\Psi(\Delta)=\left(P_{d}^{1 / 2}\right) \int_{\Delta} f(x, y) d x d y
$$

ДокАзАтельство. Первое утверждение леммы сразу вытекает из теоремы А и леммы 1. Для доказательства второго утверждения для любого $\varepsilon>0$ возьмем одновременно в качестве мажоранты и миноранты функцию $\Psi(\Delta)$. Отметим, что $\Psi\left([0,1]^{2}\right)-\Psi\left([0,1]^{2}\right)=0<\varepsilon$, т.е. условие 3$)$ из определения $\left(P_{\mathrm{d}}^{1 / 2}\right)$-интеграла выполнено. Из формулы (21) вытекает выполнение любого из условий (8)-(11) в каждой точке $(x, y) \notin[0,1]^{2} \backslash\{\widetilde{z}\}$. Для того чтобы проверить выполнение условия (11) в точке $\widetilde{z}$, для каждого $i=1, \ldots, 4$ рассмотрим $\widetilde{z}$-последовательность $\left\{\Delta_{k, l}^{i}\right\}$ такую, что $\Delta_{k, l}^{i} \subset \Omega_{i}$ для всех $k, l$. Пользуясь $(22)$, при $k, l \geqslant 1$ получаем

$$
\begin{gathered}
\Psi\left(\Delta_{n, n}^{i}\right)-\frac{1}{2} \Psi\left(\Delta_{n, n-1}^{i}\right)-\frac{1}{2} \Psi\left(\Delta_{n-1, n}^{i}\right)+\frac{1}{4} \Psi\left(\Delta_{n-1, n-1}\right) \\
=(-1)^{i-1}\left(1-\frac{1}{2} \frac{5}{4}-\frac{1}{2} \frac{5}{4}+\frac{1}{4}\right)=0 .
\end{gathered}
$$

Из (31) вытекает справедливость условия (11) в точке $\widetilde{z}$. Тем самым, все условия из определения $\left(P_{\mathrm{d}}^{1 / 2}\right)$-интеграла выполнены. Тогда формула $(30)$ вытекает из следующей цепочки, использующей (12):

$$
\Psi(\Delta) \leqslant \sup _{F_{2}} F_{2}(\Delta)=\left(P_{\mathrm{d}}^{1 / 2}\right) \int_{\Delta} f(x, y) d x d y=\inf _{F_{1}} F_{1}(\Delta) \leqslant \Psi(\Delta) .
$$

Лемма доказана. 
ЛЕмма 3. Функиия $f(x, y)$ из конструкиии 1 является $\left(H_{1, \mathrm{~d}}\right)$-интегрируемой. При этом для любого двоичного интервала $\Delta \subset[0,1]^{2} \backslash\{\widetilde{z}\}$ имеет место равенство

$$
\left(H_{1, \mathrm{~d}}\right) \int_{\Delta} f(x, y) d x d y=\Psi(\Delta)=(R) \int_{\Delta} f(x, y) d x d y .
$$

Если же $\Delta \ni \widetilde{z}$, mо $\left(H_{1, \mathrm{~d}}\right) \int_{\Delta} f(x, y) d x d y=0$.

Доказательство. Так как функция $f$ непрерывна на $[0,1]^{2} \backslash\{\widetilde{z}\}$, в силу утверждения 1 она является $\left(H_{1, \mathrm{~d}}\right)$-интегрируемой на любом двоичном квадрате $\Delta \subset$ $[0,1]^{2} \backslash\{\widetilde{z}\}$. Отсюда вытекает, с учетом (18), формула (32). Рассмотрим произвольный двоичный квадрат $\Delta \ni \widetilde{z}$, не совпадающий с $[0,1]^{2}$. Без ограничения общности считаем, что $\Delta=\Delta_{n, n}^{2^{n-1}, 2^{n-1}}$. Для всех $l=1,2, \ldots$ и $i, j=0,1$ введем обозначения

$$
U_{l}=\Delta_{l, l}^{2^{l-1}-1,2^{l-1}-1}, \quad V_{l}^{i, j}=\Delta_{l+1, l+1}^{2^{l}-2-i, 2^{l}-j}, \quad W_{l}^{i, j}=\Delta_{l+1, l+1}^{2^{l}-i, 2^{l}-2-j} .
$$

При всех $m \geqslant n$ имеет место равенство

$$
\Delta=\Delta_{m, m}^{2^{m-1}, 2^{m-1}} \cup U \cup V \cup W
$$

где

$$
U=\bigcup_{l=n+1}^{m} U_{l}, \quad V=\bigcup_{l=n+1}^{m} \bigcup_{i, j=0}^{1} V_{l}^{i, j}, \quad W=\bigcup_{l=n+1}^{m} \bigcup_{i, j=0}^{1} W_{l}^{i, j}
$$

причем двоичные квадраты из правых частей (33) и (34) попарно не перекрываются. При этом любой из квадратов из правых частей (34) не содержит точку $\widetilde{z}$. Поэтому функция $f$ является непрерывной на любом таком квадрате и в силу утверждения 1 $\left(H_{1, \mathrm{~d}}\right)$-интегрируемой на нем. Из формул (15)-(18) и (32) при всех $m>n$ вытекает следующее равенство:

$$
\sum_{l=n+1}^{m} \int_{U_{l}} f+\sum_{l=n+1}^{m} \sum_{i, j=0}^{1} \int_{V_{l}, j} f+\sum_{l=n+1}^{m} \sum_{i, j=0}^{1} \int_{W_{l}^{i, j}} f=0
$$

(под интегралом в последней формуле понимается $\left(H_{1, \mathrm{~d}}\right)$-интеграл). Пусть при $l=$ $1,2, \ldots K_{l}$ означает любой из квадратов $U_{l}, V_{l}^{i, j}$ или $W_{l}^{i, j}$. Тогда квадрат $K_{l}$ не содержит точку $\widetilde{z}$. Пусть выбрано произвольное $\varepsilon>0$. Выберем функцию $\delta: K_{l} \rightarrow$ $(0,+\infty)$ такую, что неравенство

$$
\left|\sum_{(\mathbf{z} ; I) \in P} f(\mathbf{z})\right| I\left|-\int_{K_{l}} f\right|<\frac{\varepsilon}{2^{l}}
$$

выполняется для всех $(\delta(\mathbf{z}), 1)$-разбиений $P$ квадрата $K_{l}$. Потребуем также от функции $\delta$, чтобы

$$
\delta(\mathbf{z})<\frac{1}{2} \operatorname{diam} K_{l}, \quad \delta(\mathbf{z})<\operatorname{dist}\left(\widetilde{z}, K_{l}\right) .
$$

Выбирая функцию $\delta$ для всех множеств $K_{l}$ и задав произвольным образом значение $\delta(\widetilde{z})$, получим функцию $\delta: \Delta \rightarrow(0,+\infty)$. Оговоримся, что если точка $\mathbf{z}$ принадлежит нескольким множествам $K_{l}$, то из всех значений $\delta(\mathbf{z})$ выберем минимальное. Рассмотрим произвольное разбиение $Q=\{(\mathbf{z} ; I)\}$ квадрата $\Delta$. Из (37) вытекает, что разбиение $Q$ содержит пару $(\widetilde{z}, \widetilde{I})$, где $\widetilde{I}=\Delta_{m_{0}, m_{0}}^{2_{0},^{m_{0}-1}}$ для некоторого $m_{0}>n$. 
Из (37) также вытекает, что любая другая пара $(\mathbf{z} ; I) \in Q$ обладает тем свойством, что $I \subset K_{l}$ для некоторого куба $K_{l}$. Используя эти факты, получим следующую цепочку:

$$
\begin{aligned}
\left|\sum_{(\mathbf{z} ; I) \in Q} f(\mathbf{z})\right| I|| & \stackrel{(35)}{\leqslant} \sum_{l=n+1}^{m_{0}}\left(\left|\sum_{U_{l}} f(\mathbf{z})\right| I\left|-\int_{U_{l}} f\right|+\sum_{i, j=0}^{1}\left|\sum_{V_{l}^{i, j}} f(\mathbf{z})\right| I\left|-\int_{V_{l}^{i, j}} f\right|\right. \\
& \left.+\sum_{i, j=0}^{1}\left|\sum_{W_{l}^{i, j}} f(\mathbf{z})\right| I\left|-\int_{W_{l}^{i, j}} f\right|\right) \\
& \stackrel{(36)}{\leqslant} \sum_{l=n+1}^{m_{0}}\left(\frac{\varepsilon}{2^{l}}+\frac{4 \varepsilon}{2^{l}}+\frac{4 \varepsilon}{2^{l}}\right)<9 \varepsilon .
\end{aligned}
$$

Последняя цепочка доказывает $\left(H_{1, \mathrm{~d}}\right)$-интегрируемость функции $f$ на кубе $\Delta \ni \widetilde{z}$ $\left(\Delta \neq[0,1]^{2}\right)$ и равенство нулю интеграла. Если же $\Delta=[0,1]^{2}$, то $\Delta=\bigcup_{i=1}^{4} \Omega_{i}$, откуда $\left(H_{1, \mathrm{~d}}\right) \int_{[0,1]^{2}} f=\sum_{i=1}^{4} \int_{\Omega_{i}} f=0$. Лемма доказана.

Теорема 2. $\left(P_{\mathrm{d}}^{1 / 2}\right)$-Интеграл и $\left(H_{1, \mathrm{~d}}\right)$-интеграл являются противоречивыми.

ДокАЗАТЕЛЬСтво. Пусть $f$ - функция из конструкции 1. Из (14) и (30) вытекает, что $\left(P_{\mathrm{d}}^{1 / 2}\right) \int_{\Omega_{1}} f=1$. Из леммы 3 вытекает, что $\left(H_{1, \mathrm{~d}}\right) \int_{\Omega_{1}} f=0$. Последние два равенства доказывают теорему.

Tеорема 3. Ряд $\left(S^{\star}\right)$ всюду на $[0,1]^{2} 1 / 2$-сходится $\kappa\left(H_{1, \mathrm{~d}}\right)$-интегрируемой функиии, однако $\left(S^{\star}\right)$ не является рядом Фуръе-Хаара этой функиии относительно $\left(H_{1, \mathrm{~d}}\right)$-интеграла.

ДокАЗАТЕЛЬСтво. По теореме 1 ряд $\left(S^{\star}\right)$ всюду на $[0,1]^{2} 1 / 2$-сходится к функции $f$, которая согласно лемме 3 является $\left(H_{1, \mathrm{~d}}\right)$-интегрируемой. Пусть $c_{2,2}-$ коэффициент Фурье-Хаара функции $f$ относительно $\left(H_{1, \mathrm{~d}}\right)$-интеграла. Воспользуемся определением ряда Фурье-Хаара, устройством двумерных функций Хаара и обозначениями из конструкции 1. Тогда

$$
c_{2,2}=\left(H_{1, \mathrm{~d}}\right) \int_{[0,1]^{2}} f \chi_{2,2}=\sum_{i=1}^{4}(-1)^{i-1}\left(H_{1, \mathrm{~d}}\right) \int_{\Omega_{i}} f \stackrel{\text { лемма }}{=} 30-0+0-0=0 .
$$

Таким образом, $c_{2,2}=0$. Сравним $c_{2,2}$ с $a_{2,2}-$ коэффициентом ряда $\left(S^{\star}\right)$. Воспользуемся тем, что $\left(S^{\star}\right)$ является рядом Фурье-Хаара функции $f$ относительно $\left(P_{\mathrm{d}}^{1 / 2}\right)$-интеграла. Выберем произвольную точку $\mathbf{z} \in \Omega_{1}$. Тогда

$$
\begin{aligned}
a_{2,2} & =a_{2,2} \chi_{2,2}(\mathbf{z})=\sum_{i, j=0}^{1}(-1)^{i+j} S_{2-i, 2-j}(\mathbf{z}) \\
\stackrel{(3)}{ } & \stackrel{\text { и (30) }}{=} \frac{1}{\left|\Omega_{1}\right|}\left(\Psi\left(\Omega_{1}\right)-\frac{1}{2} \Psi\left(\Omega_{1} \cup \Omega_{2}\right)-\frac{1}{2} \Psi\left(\Omega_{1} \cup \Omega_{4}\right)+\frac{1}{4} \Psi\left(\Omega_{1} \cup \Omega_{2} \cup \Omega_{3} \cup \Omega_{4}\right)\right) \\
& \stackrel{(14)}{=} \frac{1}{\left|\Omega_{1}\right|}(1-0-0+0)=4 .
\end{aligned}
$$

Таким образом, $a_{2,2}=4 \neq c_{2,2}$, что доказывает теорему. 
В заключение заметим, что доказательство теоремы 3 и предшествующих ей лемм существенно используют то, что функции Хаара определены на отрезке $[0,1]$. Часто функции Хаара рассматривают не на отрезке $[0,1]$, а на двоичной группе $G$ (см. [12; гл. 1]). При таком подходе, как несложно показать, теорема типа Дю Буа-Реймона для рядов $(1)$ будет выполняться и для $\left(H_{1, \mathrm{~d}}\right)$-интеграла, т.е. вторая часть теоремы 3 в этом случае перестает быть верной.

\section{СПИСОК ЦИТИРОВАННОЙ ЛИТЕРАТУРЫ}

[1] Н. К. Бари, Тригонометрические рядь, ГИФМЛ, М., 1961.

[2] М.Г. Плотников, "Некоторые свойства многомерных обобщенных интегралов и теоремы типа Дю Буа-Реймона для двойных рядов Хаара", Матем. сб., 198:7 (2007), 63-90.

[3] Б. С. Кашин, А.А. Саакян, Ортогональные ряды, Изд-во АФЦ, М., 1999.

[4] П. Л. Ульянов, "О рядах по системе Хаара", Матем. сб., 63:3 (1964), 356-391.

[5] W. R. Wade, K. Yoneda, "Uniqueness and quasimeasures on the group of integers of a p-series field", Proc. Amer. Math. Soc., 84:2 (1982), 202-206.

[6] В. А. Скворцов, А. А. Талалян, "Некоторые вопросы единственности кратных рядов по системе Хаара и тригонометрической системе", Матем. заметки, 46:2 (1989), $104-113$.

[7] М.Г. Плотников, "О единственности всюду сходящихся кратных рядов Хаара", Вестн. Моск. ун-та. Сер. 1. Матем., мех., 2001, № 1, 23-28.

[8] K. M. Ostaszewski, Henstock Integration in the Plane, Mem. Amer. Math. Soc., 63, 353, Amer. Math. Soc., Providence, RI, 1986.

[9] В. А. Скворцов, "О множествах единственности для многомерных рядов Хаара", Maтем. заметки, 14:6 (1973), 789-798.

[10] М. Г. Плотников, "О нарушении единственности для двумерных рядов Хаара", Вестн. Моск. ун-та. Сер. 1. Матем., мех., 2003, № 4, 20-24.

[11] А.Н. Колмогоров, А. В. Фомин, Элементы теории функиий и функиионального анализа, Классический университетский учебник, Физматлит, М., 2004.

[12] Б. И. Голубов, А. В. Ефимов, В. А. Скворцов, Ряды и преобразования Уолша. Теория и применение, Наука, М., 1987.

\section{М. Г. Плотников}

Вологодская государственная молочнохозяйственная академия им. Н. В. Верещагина

E-mail: mgplotnikov@mail.ru
Поступило

21.01.2008

Исправленный вариант

26.09.2008 